\title{
OBSERVER DESIGN FOR OPEN AND CLOSED TROPHIC CHAINS
}

\author{
Z. Varga ${ }^{a}$ M. Gámez ${ }^{b}$, I. López \\ a Institute of Mathematics and Informatics, Szent István \\ University, Páter K. u. 1., H-2103 Godollo, Hungary. (Varga.Zoltan@gek.szie.hu) \\ ${ }^{\mathrm{b}}$ Departament of Statistics and Applied Mathematics, University of Almería, \\ La Cañada de San Urbano, 04120 Almería, España. (mgamez@ual.es, \\ milopez@ual.es)
}

\begin{abstract}
Monitoring of ecological systems is one of the major issues in ecosystem research. The concepts and methodology of mathematical systems theory provide useful tools to face this problem. In many cases, state monitoring of a complex ecological system consists in observation (measurement) of certain state variables, and the whole state process has to be determined from the observed data. The solution proposed in the paper is the design of an observer system, which makes it possible to approximately recover the state process from its partial observation. Such systems-theoretical approach has been applied before by the authors to Lotka-Volterra type population systems. In the present paper this methodology is extended to a non Lotka-Volterra type trophic chain of resource - producer - primary consumer type and numerical examples for different observation situations are also presented.
\end{abstract}

Keywords: ecological monitoring, observer design, trophic chain

\section{Introduction}

The problem of sustainability of economic and social development in a broader sense also involves conservation aspects of ecology. The problem of state monitoring of population systems, even under natural conditions, is an important issue in conservation ecology. Nearly natural populations are often exposed to a strong human intervention, e.g. by wildlife management, fisheries or environmental pollution. This means that human activity may improve or break the equilibrium of the population system in question, it may also increase or decrease the genetic variability of the given populations. One of the main tasks of conservation biology is to preserve the diversity of population systems and genetic variability of certain populations. These problems make it necessary to extend the traditional approach of theoretical biology focusing only on a biological object, to the study of the system "biological object - man". This, in dynamic situation, i.e. in case of a long-term human intervention, typically requires the 
approach of mathematical systems theory (in frequently used terms, state-space modelling). On the state-space approach to modelling in population biology, Metz (1977) is an early reference, see also Metz and Dickmann (1986).

Mathematical systems theory offers a methodology to answer this question. This discipline had been developed by the 1960s to solve variety of problems faced in engineering and industry. A basic reference is Kalman et al. (1969), see also Zadeh and Desoer (1963). A recent reference is Chen et al. (2004). While by now, mathematical systems theory became quite familiar to system engineers, observability and controllability analysis of dynamic models in population biology is relatively new. In many cases, state monitoring of a complex ecological system consists in observation (measurement) of certain state variables, and the whole state process has to be determined from the observed data. In a more general setting, the state process is a system of differential equations, and instead of its concrete solution only a transform (in particular a subset of the components) of it is known (measured). The considered system is called (locally) observable, if from the observation, the underlying state process can be uniquely recovered (near an equilibrium state). Based on the a sufficient condition for nonlinear observation systems published in Lee and Markus (1971), for different coexisting Lotka-Volterra type population systems, local observability results have been obtained in part by some of the coauthors of the of the present paper in López et al. (2007a,b). Later on, in addition to these theoretical results, for Lotka-Volterra systems even a so-called observer systems has been constructed that made it possible to numerically recover the state process from the observation data, see López et al. (2007a, b), Gámez et al. (2008a,b) and Varga (2008). We also mention that, based on an observability result of Varga (1992) for nonlinear observation systems with invariant manifold, in López et al. (2008) an observer system was designed for the frequencydependent model of phenotypic observation of genetic processes.

In the present paper ecological systems of non Lotka-Volterra type will be considered. Until now in Shamandy (2005), only observability results have been obtained for systems of type resource - producer - primary consumer. In Section 2, from Shamandy (2005), the model setup and basic conditions for the existence of an equilibrium of the system are shortly recalled. Section 3 is the main body of the paper. First the theoretical background of the observer design is set up. Then the construction of the observer and the asymptotic recovery of the state process is illustrated with 
numerical examples for different observation situations. Section 4 is devoted to the discussion of the results.

\section{Description of the dynamic model}

In order to illustrate the application of the methodology of mathematical systems theory, a relatively simple food web, a trophic chain has been chosen, that in addition to populations also involves a resource (energy or nutrient). In the following, the model setup is shortly recalled from Shamandy (2005), see also Svirezhev and Logofet (1983), Jorgensen and Svirezhev (2004). For further details on trophic chains (and genaral food webs) see e.g. Odum (1971) and Yodzis (1989)

The considered model describes how a resource moves through a trophic chain. A typical terrestrial trophic chains consists in the following components:

resource, the $0^{\text {th }}$ trophic level (solar energy or inorganic nutrient), which is incorporated by

a plant population, the $1^{\text {st }}$ trophic level (producer), which transfers it to

a herbivorous animal population, the $2^{\text {nd }}$ trophic level (primary consumer).

Let it be noted that, in a longer trophic chain, the herbivores can be consumed by a predator population the $3^{\text {rd }}$ trophic level (secondary consumer), which can be followed by top predator population (tertiary consumers). In the present paper, for technical simplicity only trophic chains of the type resource - producer - primary consumer will be studied. According to the possible types of $0^{\text {th }}$ level (energy or nutrient), two types of trophic chains will be considered: open chains (without recycling) and closed chains (with recycling). At the $0^{\text {th }}$ trophic level, resource is the common term for energy and nutrient.

Let $x_{0}$ denote of the time-varying quantity of resource present in the system, $x_{1}$ and $x_{2}$, in function of time, the biomass (or density) of the producer (species 1 ) and the primary consumer (species 2), respectively. Let $Q$ be the resource supply considered constant in the model. Let $\alpha_{0} x_{0}$ be the velocity at which a unit of biomass of species 1 consumes the resource, and it is assumed that this consumption increases the biomass of this species at rate $k_{1}$. A unit of biomass of species 2 consumes the biomass of species 1 
at velocity $\alpha_{1} x_{1}$, converting it into biomass at rate $k_{2}$. Both the plant and the animal populations are supposed to decrease exponentially in the absence of the resource and the other species, with respective rates of decrease (Malthus parameters) $m_{1}$ and $m_{2}$. Finally, in a closed system the dead individuals of species 1 and 2 are recycled into nutrient at respective rates $0<\beta_{1}<1$ and $0<\beta_{2}<1$, while for an open system (where there is no natural recycling) $\beta_{1}=0, \beta_{2}=0$ holds. Then with model parameters

$$
\left.Q, \alpha_{0}, \alpha_{1}, m_{1}, m_{2}>0 ; k_{1}, k_{2} \in\right] 0,1\left[; \beta_{1}, \beta_{2} \in[0,1[\right.
$$

for the trophic chain the following dynamic model can be set up:

$$
\begin{aligned}
& \dot{x}_{0}=Q-\alpha_{0} x_{0} x_{1}+\beta_{1} m_{1} x_{1}+\beta_{2} m_{2} x_{2} \\
& \dot{x}_{1}=x_{1}\left(-m_{1}+k_{1} \alpha_{0} x_{0}-\alpha_{1} x_{2}\right) \\
& \dot{x}_{2}=x_{2}\left(-m_{2}+k_{2} \alpha_{1} x_{1}\right)
\end{aligned}
$$

Let function $f$ be defined in terms of the right-hand side of this system:

$$
f: \mathbf{R}^{3} \rightarrow \mathbf{R}^{3}, f(x)=f\left(x_{0}, x_{1}, x_{2}\right):=\left[\begin{array}{c}
Q-\alpha_{0} x_{0} x_{1}+\beta_{1} m_{1} x_{1}+\beta_{2} m_{2} x_{2} \\
x_{1}\left(-m_{1}+k_{1} \alpha_{0} x_{0}-\alpha_{1} x_{2}\right) \\
x_{2}\left(-m_{2}+k_{2} \alpha_{1} x_{1}\right)
\end{array}\right] .
$$

In Shamandy (2005), a necessary and sufficient condition were found for the existence of a non-trivial ecological equilibrium $x^{*}$ of dynamic system (2.1)-(2.3), where all components are present: system (2.1)-(2.3) has a unique equilibrium $x^{*}=\left(x_{0}^{*}, x_{1}^{*}, x_{2}^{*}\right)>0$ if and only if the resource supply is high enough, i.e.

$$
Q>Q_{2}:=\frac{m_{1} m_{2}}{\alpha_{1} k_{1} k_{2}}-\frac{\beta_{1} m_{1} m_{2}}{\alpha_{1} k_{2}} .
$$

Throughout the paper condition (2.4) will be supposed.

Remark 2.1. For $\beta_{1}>0$ the threshold $Q_{2}$ is lower than for $\beta_{1}=0$. Clearly, in the latter case the lack of recycling from species 1, a higher value of resource supply is necessary to produce the required positive equilibrium.

Remark 2.2. Moreover, this equilibrium $x^{*}$ is asymptotically stable. In order to guarantee an ecological positive equilibrium $x^{*}$ we shall suppose in this paper that condition (2.3) holds. 


\section{Construction of an observer system for a trophic chain}

For system (2.1)-(2.3) the observability (see Appendix) of it, when we observe separately each one of its variables, was proved in Shamandy (2005). Now, following the Theorem of Sundarapandian (2002) (see Appendix), we shall construct, in an explicit way, the local exponential observer for the three cases considered by Shamandy. With this aim we calculate the corresponding matrix of the linearized system of (2.1)-(2.3) at equilibrium

$$
A=\frac{\partial f}{\partial x}\left(x^{*}\right)=\left[\begin{array}{ccc}
-\alpha_{0} x_{1}{ }^{*} & -\alpha_{0} x_{0}{ }^{*}+\beta_{1} m_{1} & \beta_{2} m_{2} \\
k_{1} \alpha_{0} x_{1}{ }^{*} & 0 & -\alpha_{1} x_{1} \\
0 & k_{2} \alpha_{1} x_{2}{ }^{*} & 0
\end{array}\right] .
$$

Case 1. We consider the observation of the resources of system (2.1)-(2.3), where the observation function is

$$
h(x):=x_{0}-x_{0}^{*} \Rightarrow C:=\frac{\partial h}{\partial x}\left(x^{*}\right)=(1,0,0) .
$$

In order to construct the local observer for the considered observation system, we need to determine a matrix $H=\operatorname{col}\left(h_{1}, h_{2}, h_{3}\right)$ such that matrix $A-H C$ was Hurwitz, i.e. all its eigenvalues have negative real parts. According to the Hurwitz criterion (see e.g. Chen et al. (2004)), in terms of the normed characteristic polynomial of $A-H C$, the following necessary and sufficient condition holds:

$$
p(\lambda)=\lambda^{3}+a_{2} \lambda^{2}+a_{1} \lambda+a_{0} \text { is Hurwitz } \Leftrightarrow a_{0}, a_{1}, a_{2}>0 \text { and } a_{2} \cdot a_{1}>a_{0} .
$$

This matrix $H$ can be determined from the following theorem:

Theorem 3.1. Let us supposed that the resource supply is high enough, $Q>\frac{m_{1} m_{2}}{\alpha_{1} k_{1} k_{2}}$ and matrix

$$
H=\left(\begin{array}{c}
h_{1} \\
0 \\
1
\end{array}\right),
$$


is such that $h_{1}>\max \left\{\frac{m_{1} x_{1}^{*}}{m_{2} x_{2}^{*}}, \frac{\alpha_{0} x_{0}^{*}}{\beta_{2} k_{2}}\right\}$. Then dynamic system defined by

$$
\dot{z}=f(z)+H[y-h(z)]
$$

is a local exponential observer for system (2.1)-(2.3) with the observation of the resource (3.1).

Proof. It is sufficient to show that under the conditions of the theorem, $x^{*}$ is a Lyapunov stable equilibrium of system (2.1)-(2.3), and matrix $A-H C$ is Hurwitz. Then the proof can be concluded applying Theorem of Sundarapandian (2002) (see Appendix).

First, from $Q>\frac{m_{1} m_{2}}{\alpha_{1} k_{1} k_{2}}$ inequality $Q>Q_{2}$ also follows, which on the one hand, as quoted at the end of Section 2, implies the existence of a unique positive equilibrium. On the other hand, in Shamandy (2005), Svirezhev and Logofet it was proved, that both in open systems (with $\beta_{1}=0, \beta_{2}=0$ ) and in partially or totally closed systems (at least one of inequalities $0<\beta_{1}<1$ and $0<\beta_{2}<1$ holds) condition $Q>Q_{2}$ also implies (asymptotic) stability of the equilibrium.

From (2.1)-(2.3) the coordinates of the positive equilibrium $x^{*}$ are

$$
\begin{aligned}
& x_{0}^{*}=\frac{-\alpha_{1} Q-\frac{\beta_{1} m_{1} m_{2}}{k_{2}}+\beta_{2} m_{1} m_{2}}{-\frac{\alpha_{0} m_{2}}{k_{2}}+\alpha_{0} \beta_{2} k_{1} m_{2}}, \\
& x_{1}^{*}=\frac{m_{2}}{k_{2} \alpha_{1}}, \\
& x_{2}^{*}=\frac{-\alpha_{0} k_{1} Q+-\frac{\alpha_{0} m_{1} m_{2}}{k_{2} \alpha_{1}}-\frac{\beta_{1} k_{1} \alpha_{0} m_{1} m_{2}}{k_{2} \alpha_{1}}}{-\frac{\alpha_{0} m_{2}}{k_{2}}+\alpha_{0} \beta_{2} k_{1} m_{2}} .
\end{aligned}
$$


Now it will be proved that for the coefficients of the normed characteristic polynomial of A-HC conditions (3.2) hold. To cut short the rather tedious calculations, the following statements can be checked: Hypothesis $Q>\frac{m_{1} m_{2}}{\alpha_{1} k_{1} k_{2}} \quad$ and $\left.k_{1}, k_{2} \in\right] 0,1\left[; \beta_{1}, \beta_{2} \in\left[0,1\left[\right.\right.\right.$ imply $Q>\frac{m_{1} m_{2}}{\alpha_{1}}$ and also $\alpha_{0} x_{0}^{*}-\beta_{1} m_{1}>0$, furthermore, the latter is sufficient for $a_{1}>0$ and also used in the proof of $a_{2} \cdot a_{1}-a_{0}>0$. On the other hand,

$$
\begin{aligned}
& h_{1}>\frac{m_{1} x_{1}^{*}}{m_{2} x_{2}^{*}} \Rightarrow \alpha_{1} h_{1} k_{2} x_{2}^{*}-\beta_{1} m_{1}>0, \quad \text { to be used in the proof of } a_{0}>0 \\
& h_{1}>\frac{\alpha_{0} x_{0}^{*}}{\beta_{2} k_{2}} \Rightarrow \beta_{2} m_{2} h_{1}-\alpha_{0} \alpha_{1} x_{0}^{*} x_{1}^{*}>0 \Rightarrow a_{2} \cdot a_{1}-a_{0}>0 .
\end{aligned}
$$

From $x_{1}^{*}=\frac{m_{2}}{k_{2} \alpha_{1}}$ and $\left.k_{1}, k_{2} \in\right] 0,1\left[; \beta_{2} \in\left[0,1\left[\right.\right.\right.$ inequality $\alpha_{1} k_{2} x_{1}^{*} x_{2}^{*}-\beta_{2} k_{1} k_{2} m_{2} x_{2}^{*}>0$ can be derived, which implies $a_{0}>0$. Finally, inequalities $h_{1}, a_{0}, x_{1}^{*}>0$ directly imply $a_{2}>0$. Summing up, all inequalities conditions (3.1) hold for $p(\lambda)$. Therefore matrix $A-H C$ is Hurwitz, and concludes the proof.

Example 3.2. As a numerical example, we consider the following $Q:=10 ; \alpha_{0}:=0.3 ; \alpha_{1}:=0.1 ; \beta_{1}:=0.2 ; \quad \beta_{2}:=0.3 ; m_{1}:=0.1 ; m_{2}:=0.4 ; k_{1}:=0.5 ; k_{2}:=0.5$. In this case the considered system (2.1)-(2.3) has a positive equilibrium $x^{*}=(4.52,8,5.78)$, which is asymptotically stable (see Figure 1$)$

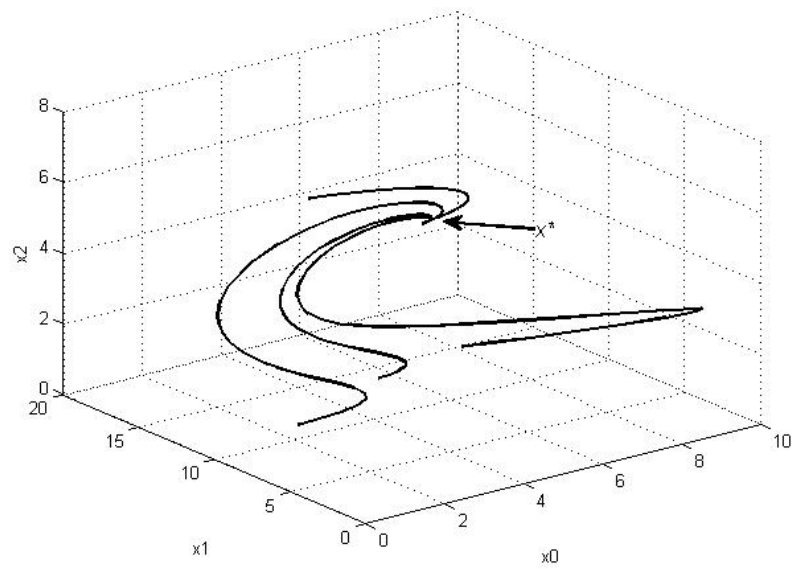

Figure 1. Some solutions of systems (2.1)-(2.3) 
and with,

$$
H=\left(\begin{array}{c}
10 \\
0 \\
1
\end{array}\right)
$$

Conditions of Theorem 3.1 are verified, therefore we can construct the following observer system

$$
\begin{aligned}
& \dot{z}_{0}=10-0.3 z_{0} z_{1}+0.2 \cdot 0.1 z_{1}+0.3 \cdot 0.4 z_{2}+10\left[y-\left(z_{0}-x_{0}^{*}\right)\right] \\
& \dot{z}_{1}=z_{1}\left(-0.1+0.5 \cdot 0.3 z_{0}-0.1 z_{2}\right) \\
& \dot{z}_{2}=z_{2}\left(-0.4+0.5 \cdot 0.1 z_{1}\right)+1\left[y-\left(z_{0}-x_{0}{ }^{*}\right)\right]
\end{aligned}
$$

If we suppose the initial condition $x_{0}:=(3,7,2)$, near the equilibrium of system (2.1)(2.3), and similarly, we consider another nearby initial condition, $z_{0}:=(2.9,7.2,1.8)$ for the observer system (3.2), Figure 2 shows that the corresponding solution $z$ tends to the solution $x$ of the original system.

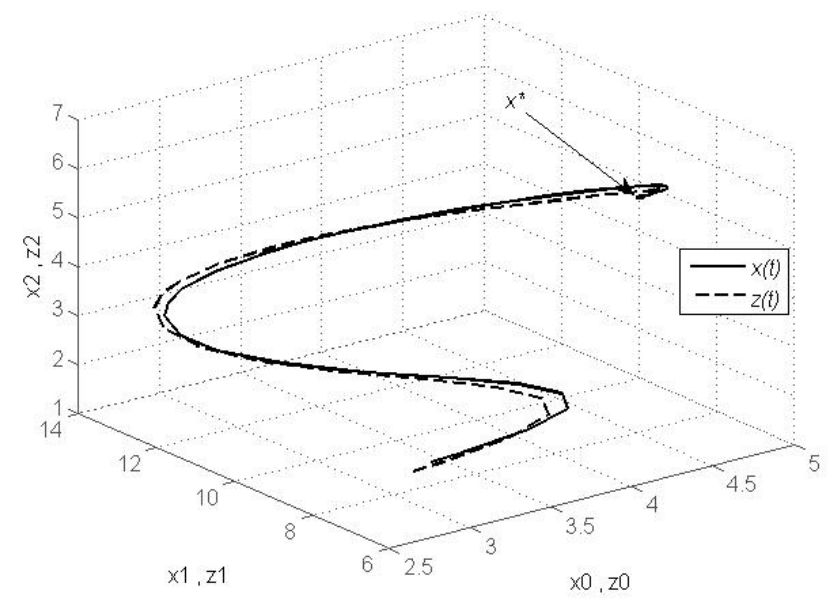

Figure 2. Solutions of systems (2.1)-(2.3) and (3.3)

Case 2. Now we consider the case when the plant of system (2.1)-(2.3) is observed. The observation function then is

$$
h(x):=x_{1}-x_{1}^{*} \Rightarrow C:=\frac{\partial h}{\partial x}\left(x^{*}\right)=(0,1,0) .
$$


Similarly to Case 1 , we can prove the following theorem providing an observer for the case (3.4).

Theorem 3.3. Given a matrix

$$
H=\left(\begin{array}{c}
h_{1} \\
h_{2} \\
0
\end{array}\right) \text {, }
$$

with $h_{1}>m_{1}$ and $h_{2}>0$, dynamic system defined by

$$
\dot{z}=f(z)+H[y-h(z)]
$$

is a local exponential observer for system (2.1)-(2.3) with the observation of the plant , as given in (3.4).

Proof. The scheme of the proof is similar to that of the previous ones. We only have to prove that matrix $A-H C$ is Hurwitz and the application Theorem of Sundarapandian (2002) will conclude the proof. Since from Shamandy (2005), $x_{1}^{*}=\frac{m_{2}}{k_{2} \alpha_{1}}$, and $\left.k_{1}, k_{2} \in\right] 0,1\left[; \beta_{2} \in\left[0,1\left[\right.\right.\right.$ we obtain that $\beta_{2} k_{1} m_{2}-\alpha_{1} x_{1}^{*}<0$. Moreover, as $\beta_{1} \in[0,1[$ and $h_{1}>m_{1}$ we have that $h_{1}-\beta_{1} m_{1}>0$. Applying these inequalities and taking into account that the case of a positive equilibrium $x^{*}>0$ is considered and $h_{2}>0$, it is easy to check that conditions (3.2) hold, therefore matrix A-HC is Hurwitz, and the proof is complete.

Example 3.4. With the same model parameters as Example 3.2, we consider

$$
H=\left(\begin{array}{c}
0.5 \\
0.1 \\
0
\end{array}\right) .
$$

Then, conditions of Theorem 3.3 are verified and therefore we can construct the following observer system

$$
\begin{aligned}
& \dot{z}_{0}=10-0.3 z_{0} z_{1}+0.2 \cdot 0.1 z_{1}+0.3 \cdot 0.4 z_{2}+0.5\left[y-\left(z_{1}-x_{1}^{*}\right)\right] \\
& \dot{z}_{1}=z_{1}\left(-0.1+0.5 \cdot 0.3 z_{0}-0.1 z_{2}\right)+0.1\left[y-\left(z_{1}-x_{1}^{*}\right)\right] \\
& \dot{z}_{2}=z_{2}\left(-0.4+0.5 \cdot 0.1 z_{1}\right)
\end{aligned}
$$

If we suppose again as initial condition $x_{0}:=(3,7,2)$, near the equilibrium of system (2.1)-(2.3), and similarly, we consider another nearby initial condition, $z_{0}:=(2.9,7.2,1.8)$ for observer system (3.5), Figure 3 shows that the corresponding solution $z$ tends to the solution $x$ of the original system. 


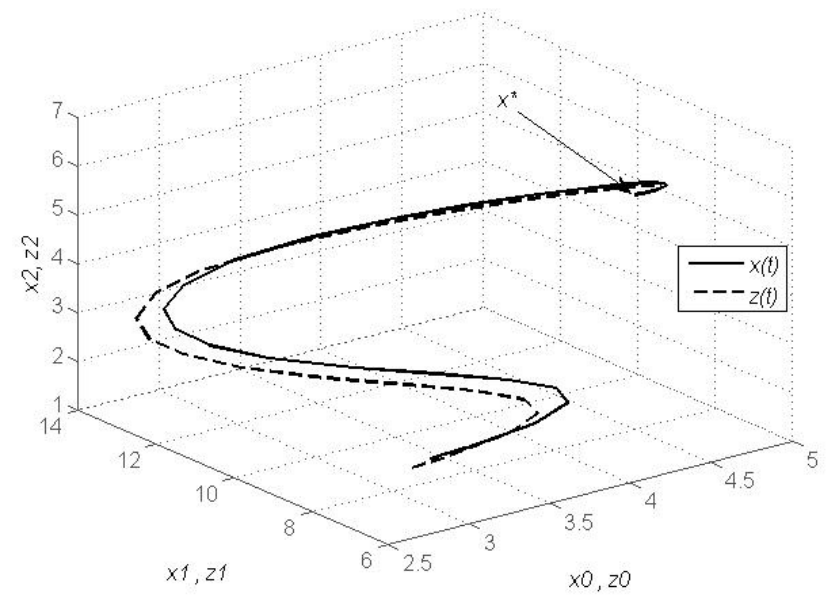

Figure 3. Solutions of systems (2.1)-(2.3) and (3.5)

Case 3. Let us finally consider the observation of the herbivorous species of system (2.1)-(2.3), where the observation function is

$$
h(x):=x_{2}-x_{2}^{*} \Rightarrow C:=\frac{\partial h}{\partial x}\left(x^{*}\right)=(0,0,1) .
$$

Similarly to Theorems 3.1 and 3.3, it is not hard to prove the following theorem providing an observer for the case (3.6).

Theorem 3.5. Given $Q>\frac{m_{1} m_{2}}{\alpha_{1} k_{1} k_{2}}$ and matrix

$$
H=\left(\begin{array}{c}
h_{1} \\
h_{2} \\
0
\end{array}\right) \text {, }
$$

where $h_{1}>m_{1}$ and $h_{2}>m_{2}$, then dynamic system defined by

$$
\dot{z}=f(z)+H[y-h(z)]
$$

is a local exponential observer for system (2.1)-(2.3) with the observation $h$ of the plant.

Example 3.6. For the model parameters of the previous examples, with

$$
H=\left(\begin{array}{c}
0.5 \\
0.5 \\
0
\end{array}\right),
$$


conditions of Theorem 3.5 hold, and hence we obtain the following observer system

$$
\begin{aligned}
& \dot{z}_{0}=10-0.3 z_{0} z_{1}+0.2 \cdot 0.1 z_{1}+0.3 \cdot 0.4 z_{2}+0.5\left[y-\left(z_{2}-x_{2}^{*}\right)\right] \\
& \dot{z}_{1}=z_{1}\left(-0.1+0.5 \cdot 0.3 z_{0}-0.1 z_{2}\right)+0.5\left[y-\left(z_{2}-x_{2}{ }^{*}\right)\right] \\
& \dot{z}_{2}=z_{2}\left(-0.4+0.5 \cdot 0.1 z_{1}\right)
\end{aligned}
$$

Set again initial condition $x_{0}:=(3,7,2)$, close to the equilibrium of system (2.1)-(2.3), and as a nearby initial condition for the observer system (3.7) also choose $z_{0}:=(2.9,7.2,1.8)$. Now Figure 4 shows that the corresponding solution $z$ tends again to the solution $x$ of the original system.

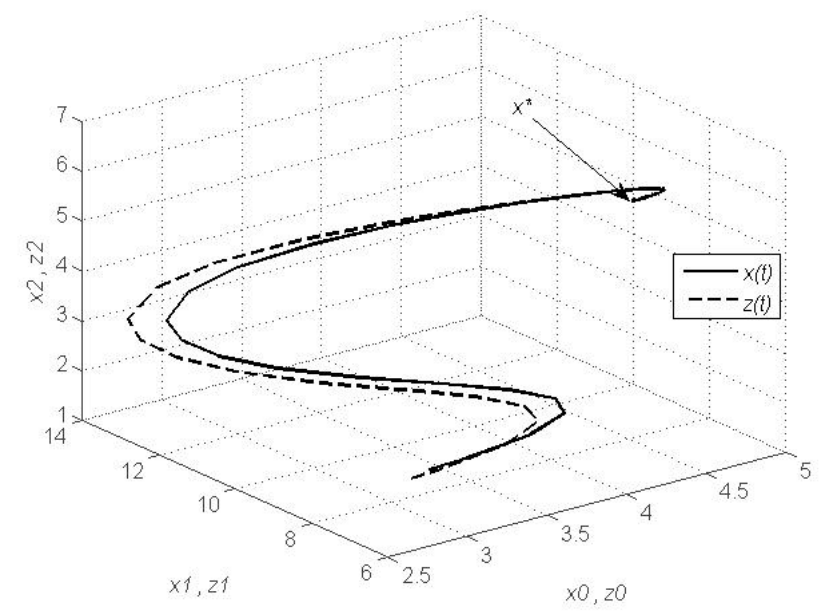

Figure 4. Solutions of systems (2.1)-(2.3) and (3.7)

\section{Discussion}

In the paper the construction of an observer system was applied for the state monitoring of a simple trophic chain of the type resource - producer - primary consumer, recovering the whole state process from the only observation of different components of the systems, such as the resource, the plant (producer) and a herbivorous animal. The applied methodology can also be extended to more complex models of food webs, involving the observation of certain abiotic environmental components and/or certain indicator species. A similar approach may be also useful for the monitoring of population systems in changing environment, where the change of certain abiotic parameters of the ecosystem is governed by an "external" dynamic system (describing an industrial pollution or climatic changes). 


\section{Acknowledgements}

The authors wish to thank the Ministry of Education and Science of Spain for the financial support of the project TIN2007-67418-C03-02, which has partially supported this work. The research was also supported by the Hungarian National Scientific Research Fund (OTKA 62000 and 68187), and a bilateral project funded by the Scientific and Technological Innovation Fund (of Hungary) and the Ministry of Education and Sciences (of Spain HH2008-0023).

\section{Appendix}

Given positive integers $m, n$, let

$$
f: \mathbf{R}^{n} \rightarrow \mathbf{R}^{n}, \quad h: \mathbf{R}^{n} \rightarrow \mathbf{R}^{m}
$$

be continuously differentiable functions and for some $x^{*} \in \mathbf{R}^{n}$ we have that $f\left(x^{*}\right)=0$ and $h\left(x^{*}\right)=0$.

We consider the following observation system

$$
\begin{aligned}
& \dot{x}=f(x) \\
& y=h(x),
\end{aligned}
$$

where $y$ is called the observed function.

Definition A.1. Observation system (A.1)-(A.2) is called locally observable near equilibrium $x^{*}$, over a given time interval $[0, T]$, if there exists $\varepsilon>0$, such that for any two different solutions $x$ and $\bar{x}$ of system (1) with $\left|x(t)-x^{*}\right|<\varepsilon$ and $\left|\bar{x}(t)-x^{*}\right|<\varepsilon(t \in[0, T])$, the observed functions $h \circ x$ and $h \circ \bar{x}$ are different. (० denotes the composition of functions. For brevity, the reference to $[0, T]$ is suppressed). For the formulation of a sufficient condition for local observability consider the linearization of the observation system (A.1)-(A.2), consisting in the calculation of the Jacobians

$$
A:=f^{\prime}\left(x^{*}\right) \text { and } C:=h^{\prime}\left(x^{*}\right)
$$

Theorem A.2. (Lee and Markus, 1971). Suppose that

$$
\operatorname{rank}\left[C|C A| C A^{2}|\ldots| C A^{n-1}\right]^{T}=n
$$


Then the observation system (A.1)-(A.2) is locally observable near equilibrium $x^{*}$.

Now, the construction of an observer system will be based on Sundarapandian (2002). Let us consider observation system (A.1)-(A.2).

Definition A.3. Given a continuously differentiable function $G: \mathbf{R}^{n} \times \mathbf{R}^{m} \rightarrow \mathbf{R}^{n}$, system

$$
\dot{z}=G(z, y)
$$

is called a local asymptotic (respectively, exponential) observer for observation system (A.1)-(A.2) if the composite system (A.1)-(A.2), (A.4) satisfies the following two requirements:

i) If $x(0)=z(0)$, then $x(t)=z(t)$, for all $t \geq 0$.

ii) There exists a neighbourhood $V$ of the equilibrium $x^{*}$ of $\mathbf{R}^{n}$ such that for all $x(0), z(0) \in V$, the estimation error $z(t)-x(t)$ decays asymptotically (respectively, exponentially) to zero.

Theorem A.4. (Sundarapandian, 2002). Suppose that equilibrium $x^{*}$ of system (A.1)(A.2) is Lyapunov stable, and that there exists a matrix $K$ such that matrix $A-K C$ is Hurwitz (i.e. its eigenvalues have negative real parts), where $A=f^{\prime}\left(x^{*}\right)$ and $C=h^{\prime}\left(x^{*}\right)$. Then dynamic system defined by

$$
\dot{z}=f(z)+K[y-h(z)]
$$

is a local exponential observer for observation system (A.1)-(A.2).

\section{References}

Chen, Ben M.; Lin, Zongli; Shamesh, Yacov A., 2004. Linear Systems Theory. A Structural Decomposition Approach. Birkhauser, Boston.

Gámez, M., López, I. and Varga, Z., 2008a?. Iterative scheme for the observation of a competitive Lotka-Volterra system. Applied Mathematics and Computation. $201811-$ 818

Gámez, M.; López, I. and Molnár, S., 2008b?. Monitoring environmental change in an ecosystem. Biosystems, 93, 211-217. 
Jorgensen, S., Svirezhev, Y. (Eds.), 2004. Towards a Thermodynamic Theory for Ecological Systems Pergamon.

Kalman, R. E., Falb, P. L., Arbib, M. A., 1969. Topics in Mathematical System Theory. McGraw-Hill, New York.

Lee, E.B. and Markus, L., 1971. Foundations of Optimal Control Theory. New YorkLondon-Sydney : Wiley.

López I, Gámez M, Molnár, S., 2007a. Observability and observers in a food web. Applied Mathematics Letters 20 (8): 951-957.

López, I., Gámez, M., Garay, J. and Varga, Z., 2007b. Monitoring in a Lotka-Volterra model. Biosystems, 83, 68-74.

López, I., Gámez, M. and Varga, Z. 2008, Observer design for phenotypic observation of genetic processes. Nonlinear Analysis: Real World Applications 9, 290-302.

Metz, J. A. J. 1977. State space model for animal behaviour. Ann. Syst. Res. 6: 65-109.

Metz, J. A. J. and Dickmann O. (Eds), 1986. The Dinamics of Physiologically structured Populations, Springer Lecture Notes in Biomath. 68.

Paginas??

Odum, E. P. 1971. Fundamentals of Ecology. 3rd ed. Saunders, Philadelphia. 574 pp.

Shamandy, A., 2005. Monitoring of trophic chains. Biosystems, Vol. 81, Issue 1, 43-48.

Sundarapandian, V., 2002. Local observer design for nonlinear systems. Mathematical and computer modelling 35, 25-36.

Svirezhev, Yu.M. and D.O. Logofet (1983). Stability of biological communities. Mir Publishers, Moscow.

Varga, Z., 1992. On Observability of Fisher's model of selection, Pure Mathematics and Applications, Ser. B. Vol. 3, No 1, 15-25.

Varga, Z., 2008. Applications of mathematical systems theory in population biology. Periodica Mathematica Hungarica. 51 (1), 157-168.

Yodzis, P. (1989). Introduction to Theoretical Ecology. Harper \& Row. New York.

Zadeh, L. A. and Desoer, C. A., 1963. Linear System Theory-The State Space Approach, New York: McGraw-Hill Book Co. 